\title{
Use of cholinesterase inhibitors in dementia
}

\author{
Mark Holden \& Cornelius Kelly
}

Alzheimer's disease is the most common cause of dementia in older people, with about half a million people affected in the UK. Its effects are devastating and far-reaching for sufferers, their carers and society in general - the Audit Commission estimated the annual cost of dementia care for 1998-1999 at $£ 6.1$ billion (Audit Commission, 2000). There is no cure and it is terminal within 3 to 7 years of diagnosis. Given an estimated doubling of dementia cases over the next 50 years (Melzer et al,1997), the burden of care is set to increase substantially.

In April 1997 donepezil hydrochloride, the first of several symptomatic drugs, was licensed in the UK. Since many health authorities refused to fund prescription of these products, the National Institute for Clinical Excellence (NICE) reviewed the evidence of their efficacy and issued formal guidance for their use (NICE, 2001).

Medication aimed at the amelioration of the underlying pathophysiology of Alzheimer's disease, with resultant improvements in the core cognitive deficits of the disorder, has, understandably, generated enthusiasm in both clinicians and patient representatives. Does the current best evidence support this, and if so, how might these new drugs be incorporated into clinical practice?

\section{Background}

For over two decades the main focus of drug development in Alzheimer's disease has been on strategies that enhance central cholinergic function. This approach has been based on the cholinergic hypothesis of Alzheimer's disease, which proposes that the decline in cognitive function is linked to the loss of cholinergic neurotransmission in the hippocampus and cortex (Perry et al, 1978).

The synthesis of acetylcholine from choline and acetyl-coenzymeA (Acetyl-coA) in presynaptic neurons is catalysed by the enzyme cholineacetyltransferase. When released, acetylcholine's effects are mediated via pre- and post-synaptic muscarinic and nicotinic receptors. Results may be either excitatory or inhibitory. Released acetylcholine is broken down within the synaptic cleft by the enzyme acetylcholinesterase (AChE).

The majority of central cholinergic activity arises in efferents from the neurons of the nucleus basalis of Meynert in the basal forebrain. These efferents project widely and similarly receive afferents from many sources.

The cholinergic hypothesis is based on many different demonstrated abnormalities within this cholinergic system. During the 1970s a 60-90\% loss of cholineacetyltransferase was demonstrated in the cerebral cortex and hippocampus of patients with Alzheimer's disease. More recently, photon emission tomography (PET) scans have demonstrated reduced activity of AChE in Alzheimer's disease. Whitehouse (1982) showed a significant loss of neurons in the nucleus basalis of Meynert of patients with Alzheimer's disease compared with age-matched controls. Consistent with this is the finding that anticholinergic drugs can produce cognitive dysfunction and confusion in otherwise healthy individuals.

\footnotetext{
Mark Holden is a specialist registrar in psychiatry at Homerton Hospital (East Wing, Homerton Hospital, London E9 6SR, UK). Cornelius Kelly is a consultant in psychiatry of the elderly at Brent, Kensington, Chelsea and Westminster Mental Health NHS Trust. Over the past decade he has developed an active interest in dementia assessment and treatment and has spoken widely on this topic.
} 


\section{Acetylcholine replacement therapy}

Acetylcholine replacement therapy has been shown to be beneficial but is likely to provide symptomatic relief only. A variety of strategies have been envisaged to implement this replacement.

\section{Acetylcholine precursors}

Loading with precursors such as choline and lecithin has been tried in several studies. Despite the use of extremely high doses, most studies failed to show significant benefit. This approach has largely been abandoned.

\section{Cholinergic agonists}

Cholinergic receptors exist in two main forms: muscarinic and nicotinic. There are distinct pre- and post-synaptic muscarinic receptors, and in Alzheimer's disease it is predominantly the presynaptic neuron that is affected. Several muscarinic agonists (e.g. xanomeline, milameline and sabcomeline) have been tried and dropped from clinical trials as either ineffective or poorly tolerated.

Nicotinic receptors are important to learning and memory, and nicotine is reported to improve attention and information-processing in Alzheimer's disease. Direct agonists tend to have their usefulness limited by vascular side-effects, but a novel approach is receptor modulation by drugs including physostigmine and galantamine. This remains an area under research.

\section{Enhancement of acetylcholinesterase release}

This approach, for example through the use of phosphatidylserine, has been attempted with little evidence of success.

\section{Acetylcholinesterase inhibition}

To date, the use of acetylcholinesterase inhibitors is the only therapy to have shown consistent positive results in the treatment of Alzheimer's disease.

There are two main types of central cholinesterase: acetylcholinesterase and butyrylcholinesterase. Acetylcholinesterase is predominant and exists in several forms. In Alzheimer's disease it seems that the extracellular (G4) form is most diminished. The enzyme has also been demonstrated in the plaques and neurofibrillary tangles that are pathological markers of the disease.

\section{Acetylcholinesterase inhibitors}

\section{Tetrahydroaminacridine ('tacrine')}

In 1993 'tacrine' was licensed in the US, Canada and parts of Europe as the first agent specifically approved for treating the cognitive symptoms of Alzheimer's disease. It is a centrally active noncompetitive reversible inhibitor of acetylcholinesterase and butyrylcholinesterase. It is also known to affect monoamine levels, bind nicotinic and muscarinic receptors and block $\mathrm{Na}^{+}$and $\mathrm{K}^{+}$. The relevance of these effects is less clear. Its clinical affects in Alzheimer's disease are modest, with 20$30 \%$ of treated patients showing improved cognitive function and functional ability after 3-6 months of treatment. This is equivalent to a delay of 6-12 months in expected cognitive deterioration. In 1994 a randomised double-blind placebo controlled parallel group study reported that only 279 of 663 $(42 \%)$ patients who entered the 30 -week trial completed it $(74 \%$ of withdrawls were due to gastrointestinal symptoms or elevated liver enzymes) (Knapp et al, 1994). A meta-analysis of tacrine trials in 1998 suggested that one patient withdrew for every four patients treated. Adverse events affected about $60 \%$ of patients; one-third of these were due to cholinergic side-effects and one-third to elevated liver transaminases (Box 1).

The frequent liver function abnormalities are due to specific reversible hepatotoxicity and make weekly monitoring of liver function tests mandatory during dose escalation. This, combined with the need to take the drug four times a day, has limited tacrine's routine clinical usefulness. It was not originally granted a UK license but was subsequently proved in 1997. The principle lessons learned from tacrine were that future acetylcholinesterase inhibitors should be less toxic, more selective and more efficacious.

\section{Box 1 Cholinergic side-effects}

Gastrointestinal symptoms: nausea, vomiting, diarrhoea

Sweating

Bradycardia

Headache 


\section{Donepezil hydrochloride}

Donepezil gained its license in March 1997 and was the first drug available in the UK for the symptomatic treatment of mild to moderate dementia in Alzheimer's disease. Donepezil is a piperidine-based reversible inhibitor of acetylcholinesterase, for which it is highly specific, with much less activity against butyrylcholinesterase (which is mainly present outside the central nervous system). Absorption of donepezil is complete and uninfluenced by either food or time of administration. This, combined with a long elimination half-life (70-80 hours) allows for once-daily dosing and improved compliance and supervision.

Initial data submitted for the registration of donepezil came from three American randomised controlled trials involving over 1000 patients for 14-30 weeks (Allen, 1999). A subsequent European multinational study of 818 patients confirmed its efficacy (Rogers et al, 1998). Primary efficacy end-points showed improvement in overall cognitive function, as measured by the Mini-Mental State Examination (MMSE; Folstein et al, 1975 ) and the cognitive subscale of the Alzheimer's Disease Assessment Scale (ADAS-Cog; Rosen et al, 1984), and in overall global effect, using the Clinicians' Interview-Based Impression of change with Carer input (CIBIC)-Plus (Reisberg \& Ferris, 1994). This semi-structured instrument examines four areas of functioning: general, cognitive, behavioural and activities of daily living. Significant improvements were noted in these primary assessments: about $26 \%$ of patients receiving $10 \mathrm{mg}$ donepezil and $15 \%$ receiving $5 \mathrm{mg}$ achieved an improvement of 7 points or more on the ADASCog (compared with $8 \%$ of those receiving placebo). This is estimated to be equivalent to a 6-12 month gain in cognitive function compared with baseline.

The symptomatic benefit of donepezil does not seem to equate with a modulation of the disease process. This is illustrated by the finding (in clinical trials) that treatment and placebo groups became indistinguishable following a 6-week placebo washout after the double-blind phase had ended (Rogers et al, 1998).

Despite its high specificity for acetylcholinesterase, donepezil continues to display class-typical side-effects, including diarrhoea, cramps, fatigue, nausea and dizziness. Fortunately these are usually mild and transient - occurring early in treatment and frequently resolving in a few days. The manufacturers urge caution in the presence of bradycardia and cardiac conduction disorders.

Clinical experience and trial data have confirmed the presence of a subgroup of patients whose response to donepezil is both more pronounced and sustained than group data suggest. Identification of the mechanism underlying this heterogeneity might provide further insight into the pre-treatment identification of potential responders.

\section{Rivastigmine}

Rivastigmine was licensed in the UK in June 1998 for the treatment of mild to moderately severe Alzheimer's disease. It is a centrally selective carbamate inhibitor of butyrylcholinesterase and acetylcholinesterase (preferentially the G1 form). Despite its short half-life (1-2 hours) it binds to and inactivates the enzyme for about 10 hours, producing a 'pseudoirreversible' inhibition. Following ingestion it is rapidly absorbed and metabolised predominantly by the liver. It is, however, only weakly proteinbound and largely unaffected by cytochrome P450 enzyme, minimising the risk of significant drug interactions. The short half-life necessitates a twice-daily dosing. Initial treatment is recommended as $1.5 \mathrm{mg}$ twice daily for 2 weeks, then $3 \mathrm{mg}$ twice daily for 2 weeks, increasing to $6 \mathrm{mg}$ twice daily if tolerated.

Rivastigmine has been subject to the largest formal clinical trial programme yet conducted for antidementia treatment (the ADENA programme). The cohort of 3300 patients was notable for its inclusion of both very old patients (nearly half were over 76 years old) and those receiving polypharmacy. Available data from the included trials suggest that patients receiving 6-12 $\mathrm{mg}$ daily achieve clinical improvements in cognition and activities of daily living similar in magnitude to those achieved with donepezil (Corey-Bloom et al, 1998). Effects seem optimal in those over 75 years old and in nonsmokers. Reported side-effects are typically related to the gastrointestinal system (e.g. nausea, vomiting and anorexia) and necessitate monitoring of patients' weight. Side-effects occur predominantly during the initial dose-titration phase. As with other cholinomimetics, caution is advised in patients with cardiac conduction defects and peptic ulcer disease.

\section{Galantamine}

Initially derived from extracts of snowdrop and daffodil bulbs, this phenonthrene alkaloid is now synthetically produced. Galantamine is a reversible competitive acetylcholinesterase inhibitor that also allosterically modulates nicotinic receptors (this effect is probably independent of its cholinesterase inhibition). Suggested, but awaiting confirmation, is the notion that this property might provide a different pattern of clinical activity to other acetylcholinesterase inhibitors.

Galantamine has an elimination half-life of about 6 hours. Metabolism produces four metabolites, one of which is more active as a cholinesterase inhibitor 
than galantamine itself. The recommended maintenance dose is $16-24 \mathrm{mg}$ daily.

Over 2000 patients have been involved in doubleblind placebo controlled trials of galantamine (Scott $\&$ Goa, 2000). Patients on the treatment arm of these studies have shown statistically significant improvements in both ADAS-Cog and CIBIC-Plus. Positive effects on cognitive symptoms have been associated with significant benefits in activities of daily living. Side-effects are class-typical and tolerability is improved with slow dose escalation.

\section{Metrifonate}

Previously used in the treatment of schistosomiasis, this pro-drug is converted to a potent irreversible acetylcholinesterase and butyrylcholinesterase inhibitor (dichlorvos). Trials have shown its highly significant beneficial effects on cognitive and noncognitive (behavioural and psychiatric disturbance) features of Alzheimer's disease (Morris et al, 1997). Despite its clearly demonstrated efficacy, trials were halted in 1998 owing to concerns about severe muscle weakness seen in some trial patients (Lamb \& Faulds, 1997).

\section{Other applications of cholinesterase inhibitors}

Although currently licensed only for use in mild to moderate Alzheimer's disease, work is in progress studying the impact of acetylcholinesterase inhibitors on cognitive impairment with other causes. Recent work has suggested that vascular lesions in the central nervous system generate cholinergic deficits, and reports of improvement using cholinesterase inhibitors in post-cerebrovascular accident patients, although anecdotal, might indicate a future role.

The profound cholinergic deficit present in dementia with Lewy bodies suggests that cholinesterase inhibitors might be of at least equal benefit in this condition as to that seen in trials with Alzheimer's disease patients (Lebert et al, 1998). Indeed, recently published data of a trial of rivastigmine in Lewy body dementia appears to support this notion (McKeith et al, 2000).

\section{Evaluating the benefits}

The demonstration of treatment benefit from cholinesterase inhibitors is fraught with difficulty.
Reasonable end-points to measure efficacy include symptomatic cognitive and behavioural improvement, combined with a slowing of disease progression and ultimately the reversal or prevention of the disease process. At present, it is clear that available therapies have been shown only to provide symptomatic relief. There remains dispute as to what might reasonably constitute a treatment benefit and how to measure it. Trials that demonstrate improvement by a few points on a cognitive function test might fail to achieve any obvious benefit regarding activities of daily living and quality of life. This issue is particularly important in Alzheimer's disease, as carer burden is more closely related to the noncognitive features of the disease. Behavioural improvement might therefore delay the move to institutional care. The Committee for Proprietary Medicinal Products has recommended the main goals of treatment in Alzheimer's disease (Box 2).

There are widespread reports of behavioural improvement during trials of cholinesterase inhibitors in patients with Alzheimer's disease and this appears to have been mirrored in routine clinical practice (McKeith et al, 2000; Mathews et al, 2000). Reduction in problem behaviour such as wandering can greatly improve a carer's quality of life and perceived ability to cope. There is as yet, however, no evidence for any effect on severe behavioural disturbance.

In interpreting the current evidence it is of value to remember that the trials have largely been funded by drug companies, who would seem unlikely to be interested in publication of findings failing to show benefit. Also, it is difficult reliably to blind such trials - as evidenced by the higher drop-out rates in treatment groups due to side-effects. The validity and reliability of instruments used to ascertain clinical improvement is occasionally dubious. For instance, the CIBIC-Plus is an impression of change and is therefore liable to be biased by a researcher's optimism. In all drug trials the effect of an inter-

\section{Box 2 Committee for Proprietary Medicinal} Products' recommended goals of treatment

Symptomatic improvement

- enhanced cognition

- increased autonomy

- improved behavioural dysfunction

Slowing or arrest of symptom progression Primary prevention of disease by intervention in key pathogenic mechanisms while presymptomatic 
vention is dependent on characteristics of the sample population and on the circumstances in which the intervention is applied. It is widely recognised that generalisation from highly selected samples to the general population is difficult. Patients in cholinesterase inhibitor trials have predominantly tended to be younger, healthier and free from complex health problems.

An American study evaluated the numbers of routine clinic patients presenting with Alzheimer's disease that would fulfil eligibility criteria for clinical trials (Schneider et al, 1997). Their estimation was that only $7.9 \%$ of the cohort would have been suitable for participation. Clearly, attempting to extrapolate findings on this sample to the ineligible $92.1 \%$ is questionable.

What is becoming evident is that cholinesterase inhibitors do have a role in alleviating the clinical symptoms of Alzheimer's disease. Choice of drug for some will be dictated by side-effects, dosing or cost, but for the majority clear guidance will be necessary in treatment decision-making by clinicians.

\section{Guidelines for use}

After licensing, there was significant variation in the prescription of cholinesterase inhibitors between different areas in the UK (a situation similar to that experienced with many other drugs). Purchasers required convincing of the usefulness of these drugs before they became part of routine clinical practice. Consequently, up to half of health authorities refused to fund them. Within 1 year of the first licenses being issued, local guidelines (e.g. Lovestone et al, 1997) were distributed. Unfortunately, it seemed that the rigidity of these frequently served merely to exclude patients from treatment. Subsequently, national guidelines for the prescription of donepezil were produced by the Standing Medical Advisory Committee (National Health Service (NHS) Executive, 1998) and, in January 2001, after extensive review of the evidence, NICE issued formal guidance (Box 3).

\section{Whom to treat}

Available data suggest that this class of drug is of most benefit to those identified in the early stages of disease. Most available trial data concern cohorts of patients with mild to moderate dementia, with a MMSE score of between 10 and 26, although severity should not be judged on MMSE score alone. For example, premorbid high IQ can hide a dementia
Box 3 Guidance from the National Institute for Clinical Excellence

Medication should be prescribed by physicians in secondary care following appropriate diagnosis

Measurements should be made at baseline and monitored every 2-4 months for response to treatment

Treatment should be continued only in those deemed to have benefited (defined as improvement or no deterioration in MiniMental State Examination score together with evidence of global improvement on the basis of behavioural and/or functional assessment

behind a score above 26 and pronounced language difficulty can lead to a spuriously low score.

How early in the disease process to commence treatment remains unknown. Commencement from diagnosis may maintain the highest level of functioning for the longest period of time but, if started too early, benefits may not easily be quantified. To this end, trials are under way of cholinesterase inhibitors in minimal cognitive impairment. Their use in people with severe dementia is controversial. Since the cholinergic deficit increases with the severity of the disease, there is potential for a greater response. However, the cost of treatment may outweigh the benefit of any improvement, particularly for people in residential or nursing care. Interestingly, there is some evidence that patients with one or more copies of the APOE4 allele, coding for the apolipoprotein $\mathrm{E}$ (ApoE) variant $\varepsilon 4$, are less likely to respond to these drugs. The clinical significance of this finding remains to be demonstrated (Selkoe, 1997).

As with the initiation of any treatment, special attention must be paid to ensuring that the patient and his/her carers are aware of the diagnosis, prognosis, treatment options (including their limitations) and side-effects. Informed consent and the agreement of an overall treatment plan in the initial stages of contact are essential (Box 4).

\section{Who should prescribe?}

Current guidelines suggest that the prescription of cholinesterase inhibitors and monitoring of their effects should be provided by specialist dementia 
Box 4 Initiating treatment with acetylcholinesterase inhibitors

1. Interview patient and caregiver and establish diagnosis of probable Alzheimer's disease using standardised criteria (e.g. DSM-IV or ICD-10)

2. Document the cognitive, functional and behavioural deficits; perform baseline physical (including neurological) examination

3. Estimate stage of disease (e.g. mild, moderate or severe) using clinical skills or formalised instruments

4. Discuss diagnosis, prognosis and treatment options with patient and caregiver

5. Agree overall treatment plan

services. Pressure has therefore arisen from purchasers of health care to expand these services in terms of finance as well as appropriately trained staff. As clinical experience with cholinesterase inhibitors increases, it can be envisaged that treatment and monitoring is likely to be shifted at least to some extent towards primary care. At present, there remains a need for close liaison between primary and secondary care services in order to ensure that all those who might potentially benefit from treatment initiation are brought to specialist attention.

It is apparent that new symptomatic treatments must be considered as only one part of a wider care package aimed at optimising patient functioning and safety and caregiver support.

\section{Assessment of response}

Monitoring of clinical response to treatment is, of course, mandatory. All treatments need to be shown to be of benefit to each patient. For most drugs this is achieved by report from the patient or the carer that treatment is both helpful and tolerable. Since cholinesterase inhibitors have yet to be shown to modify the disease process itself, and the natural history of Alzheimer's disease is that of a progressive condition with periodic stabilisation, potential areas of benefit need to be clearly identified before treatment is initiated and baseline measurements recorded.

Cognition is the area most commonly assessed, although this may be of less importance to the patient or carer than non-cognitive, behavioural and functional symptoms. The MMSE and clockdrawing tests (Agrell \& Dehlin, 1998) are both quick and relatively simple screening tests for dementia and recording change over time. Measurement of behavioural and functional deterioration as well as overall clinical impression are equally important. Alzheimer's disease usually presents at a time when basic functions (e.g. feeding, dressing and selfcare) are still relatively intact, but more complex 'instrumental' activities of daily living (e.g. using the telephone, managing finances) are impaired. Clinical trials have tended to use complex measures of these, which are not readily transposed to the clinical setting, but scales have been developed that are appropriate for routine use (e.g. the Bayer-ADL; Hindmarsh et al, 1998).

Behavioural and neuropsychiatric symptoms are common and often precipitate the move to institutional care. The Neuropsychiatric Inventory (Cummings et al, 1994) assesses 12 behaviours that focus on key areas such as apathy, hallucinations and agitation.

Assessments are increasingly including an account of carer stress - both through clinical impression formed at interview and through the use of more formal instruments (Vitiliano \& Young, 1991).

Treatment should be assessed for side-effects at 4-6 weeks after initiation and subsequently at intervals of about 3 months for symptom modification or patient improvement. The decision to stop treatment is usually made on the basis of failure to respond, medication not being tolerated, noncompliance or if the treatment no longer provides benefit. Clinical experience suggests that efficacy appears to start decreasing after about 1 year of treatment, although some patients continue to benefit for much longer. Difficulties arise when the patient loses capacity to consent or is moved to institutional care. Both situations frequently prompt a discontinuation of treatment. The decision to stop is not always straightforward, particularly if it is difficult to establish a response or if a discrepancy exists between carer report and clinical findings.

\section{Potential impact of cholinesterase inhibitors}

The modest effect of currently available cholinesterase inhibitors in Alzheimer's disease falls well short of a cure. They do, however, offer considerable hope for the future.

Any new treatment that could delay the onset of Alzheimer's disease will reduce its prevalence, owing to mortality from other causes. A delay of only 1 month in admission to a nursing home has been equated with the cost of 1 year's supply of currently 
licensed treatment. A direct effect on cognitive decline and behavioural problems increases the willingness of caregivers to continue caring and thus delays the need for residential care. Often admission is precipitated by a combination of factors such as the exhaustion of carer resources - both emotional and financial.

If life expectancy is unchanged by treatment (which is currently under research) it becomes likely that patients will spend a smaller proportion of their life after diagnosis in long-term care. As a consequence, the cost of treatment itself might be reduced. However, available cholinesterase inhibitors currently cost up to $£ 1200$ per patient per annum and there is clearly a considerable cost involved in specialist assessment and monitoring - current provision of which could easily be overwhelmed if the rate of referral were markedly to increase. The new cholinesterase inhibitors may therefore not reduce the cost of Alzheimer's disease to the NHS. However, even if it is cost-neutral, the slowing of cognitive decline and loss of functioning clearly has great potential to improve the quality of life for patients and their carers.

Not all patients with dementia have Alzheimer's disease at a stage appropriate for treatment with cholinesterase inhibitors. Indeed, many that do will not wish to receive treatment because of either tolerability or personal choice. However, that the treatment is available to suitable patients seems a justifiable cost that the NHS must bear. It would seem likely that the cholinesterase inhibitors will have applications beyond their current use in dementia in Alzheimer's disease. Future use will require demonstrable advantages at both the trial stage and clinical audit. As experience grows with this class of drug, their prescription will increasingly become the concern of primary care. It will be necessary to develop protocols by which primary and secondary care services can integrate the effective provision and monitoring of treatment. The additional time this will require, together with the expertise necessary in cognitive assessment, might see the attachment of specialist liaison nurses to primary care, supervised at secondary care level.

The cost of these interventions seems a relatively low price to pay if they can diminish the human tragedy of Alzheimer's disease. The least sufferers might expect is parity with the restrictions placed on the introduction of other potentially beneficial treatments.

\section{References}

Agrell, B. \& Dehlin O. (1998) The clock-drawing test. Age and Ageing, 27, 399-403.
Allen, H. (1999) Anti-dementia drugs. International Journal of Geriatric Psychiatry, 14, 239-243.

Audit Commission (2000) Forget Me Not. Mental Health Services of Older People. London: Audit Commission.

Selkoe, D. J. (1997) Alzheimer's disease: genotypes, phenotypes and treatments. Science, 275, 630-631.

Corey-Bloom, J., Anand, R. \& Veitch, J. (1998) A randomised trial evaluating the efficacy and safety of rivastigmine in patients with mild to moderately severe Alzheimer's disease. International Journal of Geriatric Psychopharmacology, $1,55-65$

Cummings, J. L., Mega, M., Gray, K., et al (1994) The neuropsychiatric inventory: comprehensive assessment of psychopathology in dementia. Neurology, 44, 2308-2314.

Folstein, M. F., Folstein, S. E. \& McHugh, P. R. (1975) “MiniMental State": a practical method for grading the cognitive state of patients for the clinician. Journal of Psychiatric Research, 12, 189-198.

Hindmarsh, I., Lehfeld, H. de Jongh, P., et al (1998) The Bayer Activities of Daily Living Scale (B-ADL). Dementia and Geriatric Cognitive Disorders, 9(suppl. 2), 20-26.

Knapp, M. J., Knopman, D. S., Solomon, P. R., et al (1994) A 30 week randomised control trial of high dose tacrine in patients with Alzheimer's disease. JAMA, 271, 985-991.

Knopman, D., Knapp, M., Gracon, S., et al (1994) The Clinician Interview-Based Impression (CIBI). Neurology, 44, 2315-2321.

Lamb, H. M. \& Faulds, D. (1997) Metrifonate. Drugs and Aging, 11, 490-496.

Lebert, F., Pasquier, F. \& Petit, H. (1998) Tacrine efficacy in Lewy body dementia. International Journal of Geriatric Psychiatry, 13, 516-519.

Lovestone, S., Graham, N., Howard, R., et al (1997) Guidelines on drug treatment for Alzheimer's disease. Lancet, 350, 232-233.

McKeith, I., Del Ser, T., Spano, P. F., et al (2000) Efficacy of rivastigmine in dementia with Lewy bodies. A randomised, double-blind, placebo-controlled international study. Lancet, 356, 2031-2036.

Mathews, H. P., Korbey, J., Wilkinson, D. G., et al (2000) Donepezil in Alzheimer's disease: eighteen month results from the Southampton Memory Clinic. International Journal of Geriatric Psychiatry, 15, 713-720.

Melzer, D., Ely, M. \& Brayne, C. (1997) Cognitive impairment in elderly people: population based estimate of the future in England, Scotland and Wales. BMJ, 315, 462.

Morris, J., Cyrus, P., Orazem, J., et al (1997) Effects of Metrifonate on the cognitive, global and behavioral function of Alzheimer's disease patients: results of a randomised double-blind placebo controlled study. Neurology, 48, 1730.

NHS Executive (1998) SMAC Guidelines to Clinicians on the use of Donepezil for Alzheimer's Disease. London: NHS Executive.

National Institute for Clinical Excellence (2001) Guidance on the use of donepezil, rivastigmine and galantamine for the treatment of Alzheimer's disease. NICE Technology Appraisal Guidance No. 19 (http://www.nice.org.uk/pdf/ ALZHEIMER_full_guidance.pdf).

Perry, E. K., Tomlinson, B. E., Blessed, G., (1978) Correlation of cholinergic abnormalities with senile plaques and mental test scores in senile dementia. BMJ, 2, 1457-1459.

Reisberg, B. \& Ferris, S. H. (1994) CIBIC-Plus Interview Guide. East Hannover, NJ: Sandoz Pharmaceutical Corporation.

Rogers, S. L., Farlow, M. R., Doody, R. S., et al (1998) A 24 week double-blind placebo controlled trial of donepezil in patients with Alzheimer's disease. Neurology, 50, 136145

Rosen, W. G., Mohs, R. G. \& Davis, K. L. (1984) A new rating scale for Alzheimer's disease. American Journal of Psychiatry, 141, 1356-1364.

Schneider, L. S., Olin, J. T., Lyness, S. A., et al (1997) Eligibility of Alzheimer's disease clinic patients for clinical trials. Journal of the American Geriatric Society, 45, 923-928.

Scott, L. J. \& Goa, K. L. (2000) Galantamine - a review of its use in Alzheimer's disease. Drugs, 60(5), 1095-1122. 
Vitiliano, P. P. \& Young, H. M. (1991) Burden: a review of measures used among care givers of individuals with dementia. Gerontologist, 31, 67-75.

Whitehouse, P. J., Price, D. L., Strubie, R. G., et al (1982) Alzheimer's disease and senile dementia: loss of neurons in the basal forebrain. Science, 215, 1237-1239.

\section{Multiple choice questions}

1. NICE guidance on the use of donepezil, rivastigmine and galantamine states that:

a treatment should be initiated by a specialist

b efficacy or response to treatment should be assessed at 6 months

c patients with dementia with Lewy bodies may also be given these drugs

d it is necessary to show an improvement in the MMSE score for treatment to be continued

e the guidance applies only to patients with Alzheimer's disease.

2. Alzheimer's disease:

a is the most common cause of dementia in older people

b affects one million people in the UK

c causes a profound loss of cholinergic transmission

d can be slowed down by cholinesteraseinhibiting drugs

e is diagnosed using the MMSE.

3. The cholinergic hypothesis states that:

a there is a profound loss of central serotonin activity

b cognitive impairment and memory loss are due to a reduction in cholinergic transmission in subcortical structures

c released acetylcholine is broken down in the synaptic cleft by acetylcholinesterase

$\mathrm{d}$ the synthesis of the neurotransmitter is catalysed by the enzyme cholineacetyltransferase

e the majority of central cholinergic activity arises in efferents from the nucleus basalis of Meynert.
4. With regard to the use of acetylcholinesterase inhibitors:

a tetrahydroaminoacridine (tacrine) was licensed in the USA in the early 1990s as a treatment for Alzheimer's disease

b tacrine has limited use because of its cardiotoxicity

c they should be used with caution in patients with a history of peptic ulcer disease

$\mathrm{d}$ they inhibit both acetyl- and butyrylcholinesterase enzymes

e gastrointestinal disturbance is a class effect.

5. Donepezil hydrochloride:

a is a non-reversible inhibitor of acetylcholinesterase

b has a short half-life

c causes hepatotoxicity

d has a high specificity for acetylcholinesterase

e is taken once daily.
MCQ answers

$\begin{array}{llllll}1 & 2 & 3 & 4 & 5\end{array}$

a $\mathrm{T}$ a $\mathrm{T}$ a $\mathrm{F}$ a $\mathrm{T}$ a $\mathrm{F}$

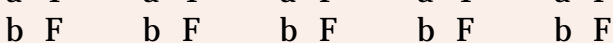

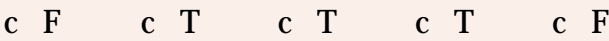

$\begin{array}{llllll}\text { d F } & d \mathrm{~F} & \mathrm{~d} T & \mathrm{~d} \mathrm{~T} & \mathrm{~d} T\end{array}$

e $\mathrm{T}$ e $\mathrm{F}$ e $\mathrm{T}$ e $\mathrm{T}$ e $\mathrm{T}$

